\title{
Long-term treatment with valganciclovir improves lentiviral suicide gene therapy of glioblastoma
}

\author{
Jubayer A. Hossain, Md A. Latif, Lars A. R. Ystaas, Sandra Ninzima, Kristoffer Riecken, \\ Arnaud Muller, Francisco Azuaje, Justin V. Joseph, Krishna M. Talasila, Jiwan Ghimire, \\ Boris Fehse, Rolf Bjerkvig, and Hrvoje Miletic
}

\begin{abstract}
Department of Biomedicine, University of Bergen, Bergen, Norway (J.A.H., L.A.R.Y., M.A.L., S.N., J.V.J., K.M.T., J.G., R.B., H.M.); Department of Pathology, Haukeland University Hospital, Bergen, Norway (J.A.H., S.N., M.A.L., H.M.); Research Department Cell and Gene Therapy, Department of Stem Cell Transplantation, University Medical Center, Hamburg, Germany (K.R., B.F.); Bioinformatics Team, Center for Quantitative Biology, Luxembourg Institute of Health, Strassen, Luxembourg (A.M., F.A.); Norlux Neuro-Oncology Laboratory, Department of Oncology, Luxembourg Institute of Health, Strassen, Luxembourg (R.B.)
\end{abstract}

Corresponding Author: Hrvoje Miletic, MD, PhD, Department of Pathology, Haukeland University Hospital, Jonas Lies Vei 65, 5021 Bergen, Norway (Hrvoje.miletic@uib.no)

\begin{abstract}
Background. Suicide gene therapy for malignant gliomas has shown encouraging results in the latest clinical trials. However, prodrug application was most often restricted to short-term treatment (14 days), especially when replication-defective vectors were used. We previously showed that a substantial fraction of herpes simplex virus thymidine kinase (HSV-TK) transduced tumor cells survive ganciclovir (GCV) treatment in an orthotopic glioblastoma (GBM) xenograft model. Here we analyzed whether these TK+ tumor cells are still sensitive to prodrug treatment and whether prolonged prodrug treatment can enhance treatment efficacy.

Methods. Glioma cells positive forTK and green fluorescent protein (GFP) were sorted from xenograft tumors recurring after suicide gene therapy, and their sensitivity to GCV was tested in vitro. GBM xenografts were treated with HSV-TK/GCV, HSV-TK/valganciclovir (valGCV), or HSV-TK/valGCV + erlotinib. Tumor growth was analyzed by $\mathrm{MRI}$, and survival as well as morphological and molecular changes were assessed.

Results. TK-GFP+ tumor cells from recurrent xenograft tumors retained sensitivity to GCV in vitro. Importantly, a prolonged period $(3 \mathrm{mo}$ ) of prodrug administration with valganciclovir (valGCV) resulted in a significant survival advantage compared with short-term ( $3 \mathrm{wk}$ ) application of GCV. Recurrent tumors from the treatment groups were more invasive and less angiogenic compared with primary tumors and showed significant upregulation of epidermal growth factor receptor (EGFR) expression. However, double treatment with the EGFR inhibitor erlotinib did not increase therapeutic efficacy.

Conclusion. Long-term treatment with valGCV should be considered as a replacement for short-term treatment with GCV in clinical trials of HSV-TK mediated suicide gene therapy.
\end{abstract}

\section{Key Points}

1. The prodrug administration scheme is a crucial factor in suicide gene therapy.

2. Prolonged prodrug administration results in improved survival.

Glioblastoma (GBM) is the most aggressive primary brain tumor in humans. Standard therapy that involves maximal possible surgical resection followed by concomitant temozolomide chemotherapy and radiotherapy results in a median survival of 14.6 months. ${ }^{1}$ Intrinsic heterogeneity ${ }^{2}$ and local seclusion provided by the blood-brain barrier (BBB), combined with the infiltrative nature, make it difficult to develop targeted therapeutics for GBM. 


\section{Importance of the Study}

Clinical trials for suicide gene therapy of GBM with the HSV-TK/GCV system used short-term prodrug treatment (14 days) as a standard regimen for suicide induction. In an orthotopic patient-derived xenograft (PDX) model for GBM we demonstrate that a substantial fraction of tumor cells expressing the suicide gene HSV-TK survive short-term treatment with the prodrug ganciclovir. We verify that these tumor cells are not resistant to treatment, but retain sensitivity to GCV in vitro. We show that treatment with valganciclovir, an alternative prodrug allowing oral long-term administration, significantly prolongs survival in a clinically relevant orthotopic PDX model. Thus, future clinical trials should consider long-term prodrug treatment to enhance the therapeutic effect of suicide gene therapy for glioblastoma.
Fairly efficient access of various prodrugs through BBB after systemic delivery has turned suicide gene therapy into a promising option for GBM treatment. Early clinical trials using replication-defective retroviral vectors encoding the suicide gene herpes simplex virus thymidine kinase (HSV-TK) to transduce GBM cells followed by ganciclovir (GCV) application were disappointing, most likely due to low transduction efficiency. ${ }^{3-6}$ One major drawback of retroviral vectors is the incapability of transducing quiescent cells. In contrast, lentiviral vectors efficiently transduce quiescent cells, mediated by the karyophilic nature of the lentiviral pre-integration complex. ${ }^{7}$ Indeed, we observed significantly higher in vitro transduction rates of biopsyderived and cancer stemlike glioma cells compared with retroviral vectors. ${ }^{8}$ We also showed that normal brain cells transduced by HSV-TK are not killed by GCV prodrug treatment ${ }^{9}$ but nonetheless can contribute to eliminating tumor cells through the bystander effect. ${ }^{10}$ By using lentiviral suicide gene therapy in a biopsy-derived GBM xenograft model, we demonstrated complete remission of GBM on MRI with a significant survival benefit. ${ }^{8}$ However, tumor recurrence was observed in all animals after termination of GCV administration. Importantly, we observed that a fraction of HSV-TK transduced tumor cells survived even after 4 weeks of GCV application. ${ }^{8}$ At this point it was not known if HSV-TK in these tumor cells retained functionality. In the present study, we show that the HSV-TK+ recurrent tumor cells harbor functional HSV-TK, which indicates that the duration of GCV application likely has not been long enough to efficiently target the slowly dividing or relatively quiescent glioma cells. By using valganciclovir (valGCV) as a prodrug, we confirmed our hypothesis showing significantly increased survival after long-term prodrug administration.

\section{Materials and Methods}

\section{Ethics Statement}

The Norwegian Data Inspectorate and the Regional Committee for Ethics in Research have approved this project (REK 013.09). The study was performed in accordance with the Helsinki Declaration.

\section{Cells and Spheroids}

Biopsy material was obtained from resection surgery of GBM patient $\# 3^{11}$ in Haukeland University Hospital according to a similar protocol mentioned previously. ${ }^{12,13}$ Resultant GBM spheroids were passaged in vivo by serial implantation in Rowett nude (RNU) rats. Details of culture condition and generation of spheroids are described in the Supplementary Methods. Spheroids were implanted as described previously. ${ }^{8}$

\section{Animals}

Healthy RNU rats were procured and provided with standard pellet diet and water ad libitum. Animals were treated in accordance with the Norwegian Animal Act. The local ethical committee approved the protocol.

\section{Lentiviral Vector Construction, Production, and Injection}

Lentiviral vectors were constructed, prepared, and injected intratumorally following protocols reported previously. ${ }^{14}$

\section{MRI}

Details of brain MRI sequences have been reported previously. ${ }^{14}$

\section{Treatment}

GCV (Cymeven; F. Hoffmann-La Roche) was given $50 \mathrm{mg} / \mathrm{kg} /$ day i.p. for 3 weeks, and valGCV (Valcyte; F. Hoffmann-La Roche) was given $80 \mathrm{mg} / \mathrm{kg} /$ day p.o. for either 3 months or in cycles starting from 5 days after vector infusion. Erlotinib (Tarceva; F. Hoffmann-La Roche) was dissolved in acidified water $(\mathrm{pH} 4.8)$, mixed at a 1:1 ratio with $0.2 \%$ carboxymethylcellulose, and given p.o. $20 \mathrm{mg} /$ $\mathrm{kg} /$ day for 5 days a week. This dosage resulted in side effects manifested by rash and weight reduction and led us to reduce the dose after 1 week to $5 \mathrm{mg} / \mathrm{kg} /$ day for 5 days a week, which did not further result in any detectable side effects.

\section{Immunohistochemistry, Immunofluorescence,} and Digital Analysis

Immunohistochemistry of paraffin sections was performed as described previously. ${ }^{8}$ Immunofluorescence was performed as described in the Supplementary Methods. 
Dilution of primary antibodies is described in the Supplementary Methods. Images were acquired with a Nikon E600 light microscope, NanoZoomer XR scanner (Hamamatsu Photonics), and Nikon widefield fluorescent microscopeTE2000.

Digital analysis of images is described in the Supplementary Methods.

\section{Statistics}

Survival analysis was performed by Kaplan-Meier estimator with log rank test using GraphPad Prism software. Other statistical analyses were performed by Stata software or $\mathrm{R}$ (RStudio v1.1.383) by $t$-test or ANOVA ( $P<0.05$ was considered significant). Significant ANOVA results were followed by post-hoc pairwise comparisons with either Tukey's or Dunnett's adjustment for multiple comparisons. In the case of heteroscedasticity, dependent variables were log or square-root transformed, depending on suitability.

\section{Gene Expression Data Analysis}

Whole-genome RNA sequencing was carried out at Macrogen (South Korea) on a NovaSeq 6000 System (Illumina). Details are described in the Supplementary Methods.

\section{Results}

\section{TK+ Glioma Cells that Survive Short-Term Prodrug Administration Retain Sensitivity Toward GCV In Vitro}

Previously we have shown that a fraction of tumor cells expressing HSV-TK survive GCV prodrug treatment and are present in recurrent tumors. ${ }^{8}$ These cells either may have become resistant to prodrug treatment or may be more quiescent than other tumor cells in vivo. Here we investigated whether these remaining TK-transduced cells retain or lose sensitivity toward GCV. Intratumoral injection of lentiviral vectors encoding TK.007, ${ }^{14}$ a recombinant HSV-TK with superior functionality, ${ }^{15,16}$ followed by GCV administration for 3 weeks resulted in tumor remission on MRI (Supplementary Fig. 1A). Recurrent tumors, which developed 7-8 weeks later, were dissociated and maintained in short-term culture. TK+ cells were sorted from the cultures based on expression of enhanced green fluorescent protein (eGFP), and immunostaining for human nestin confirmed that these cells were tumor cells of human origin (Fig. 1A). Treatment of the sorted TK+ glioma cells with GCV in vitro showed that the cells retained sensitivity toward GCV (Fig. 1B). Upon further characterization of the culturedTK+ andTK- glioma cells from the recurrent tumors, we observed no difference in terms of expression of glioma stem cell markers such as cluster of differentiation (CD)133, sex determining region $Y$-box 2, and nestin (Supplementary Fig. 1B). Similarly, there was no indication of a switch toward a mesenchymal or proneural subtype by using YKL40 (chitinase 3-like protein 1) or oligodendrocyte transcription factor 2 (olig2) markers, respectively (Supplementary Fig. 1B). In conclusion, TK+ glioma cells do not become resistant to prodrug treatment over time.

\section{Long-Term valGCV Administration Improves the Therapeutic Effect of Suicide Gene Therapy for Experimental GBM}

The sustained GCV sensitivity of the TK+ cells indicated that the current prodrug scheme of 2-3 weeks of GCV treatment, which was also used in clinical trials for suicide gene therapy, 6,17 is too short to eliminate all transduced tumor cells. To assess the effect of prolonged prodrug administration we introduced the drug valGCV, which is metabolized into GCV in the intestinal wall and liver. ${ }^{18}$ ValGCV is given orally and thus is suitable for long-term administration. ${ }^{19}$ In contrast, GCV is given i.v. to patients by using catheters, which can cause infections upon longterm use. ${ }^{20}$ We compared the therapeutic benefit of longterm valGCV administration (3 mo) with short-term (3 wk)
A

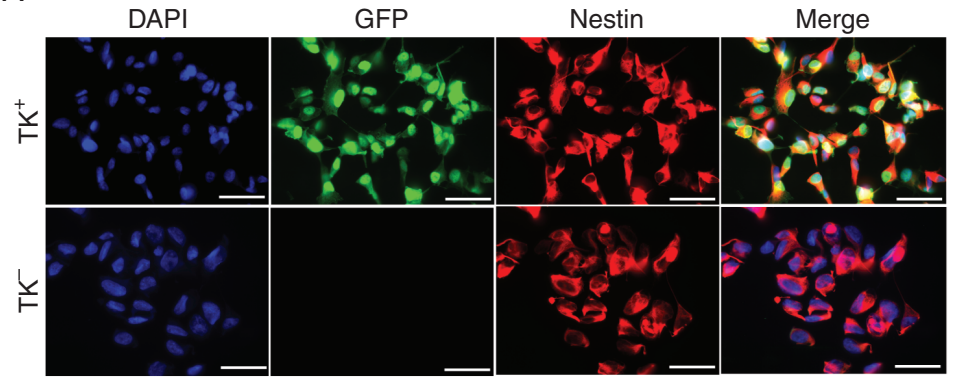

B

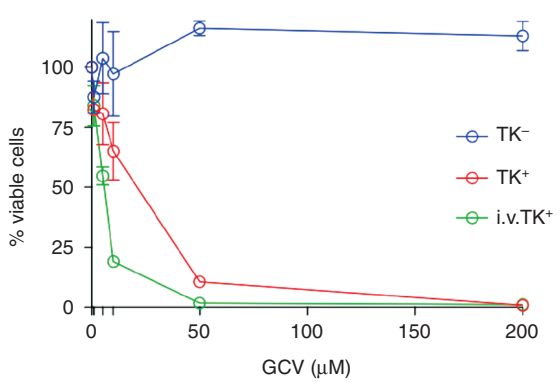

Fig. 1 TK+ glioma cells that survive short-term prodrug administration retain sensitivity to GCV in vitro. (A) Immunofluorescence staining with antibodies against human nestin (red) and GFP (green). Scale bar: $50 \mu \mathrm{m}$. (B) Cell viability assay showing percent of surviving cells after increasing concentrations of GCV; i.v. TK+: The TK- cells present in the recurrent tumor were transduced with the same lentiviral vector in vitro. 
GCV treatment in the same orthotopic xenograft model. During treatment, the majority of animals from both treatment groups showed rapid reduction in tumor growth followed by tumor remission on MRI within 3-4 weeks (Fig. 2A). Kaplan-Meier survival analyses revealed that the TK/valGCV treatment group had a significant survival benefit compared with the TK/GCV group (Fig. 2B). Interestingly one animal from the valGCV treatment group was apparently cured and sacrificed healthy after more than 6 months of tumor remission (Supplementary Fig. 2A). All other animals in the valGCV treatment group developed recurrences distant from the primary site and often close to or in the brainstem (Supplementary Fig. 2B), which might explain why the difference in survival between the 2 treatment groups was not more extensive.

\section{Continuous valGCV Application Eliminates the Majority of TK+ Tumor Cells}

The majority of animals demonstrated tumor recurrence at various time periods after initial remission on MRI. While in the GCV treatment group 4 out of 4 animals showed local recurrences, all animals in the valGCV group showed recurrences that were distant from the primary site, indicating that highly invasive cells escaped the treatment (Fig. 3A). Next, we analyzed whetherTK-transduced cells had survived long-term treatment with valGCV. While TK-transduced cells were abundant in the recurrences of GCV-treated animals, the distant recurrences of valGCV-treated animals were negative for the TK transgene. However, scattered TK+ cells were detected at the primary site of valGCV-treated animals (Fig. 3B). When quantifying the number of transduced tumor cells on double immunofluorescence stained sections with antibodies against human nestin and GFP (Fig. 3C), we identified a significantly lower number of transduced residual glioma cells in the valGCV group compared with the GCV group (Fig. 3D). Further, we observed that the residual $\mathrm{TK}+$ cells in the short-term GCV group are significantly less proliferative than the TK- cells (Fig. 3E). We conclude that prolonged valGCV application facilitates the elimination of slow-proliferating glioma cells that are spared by current short-term treatment regimen.

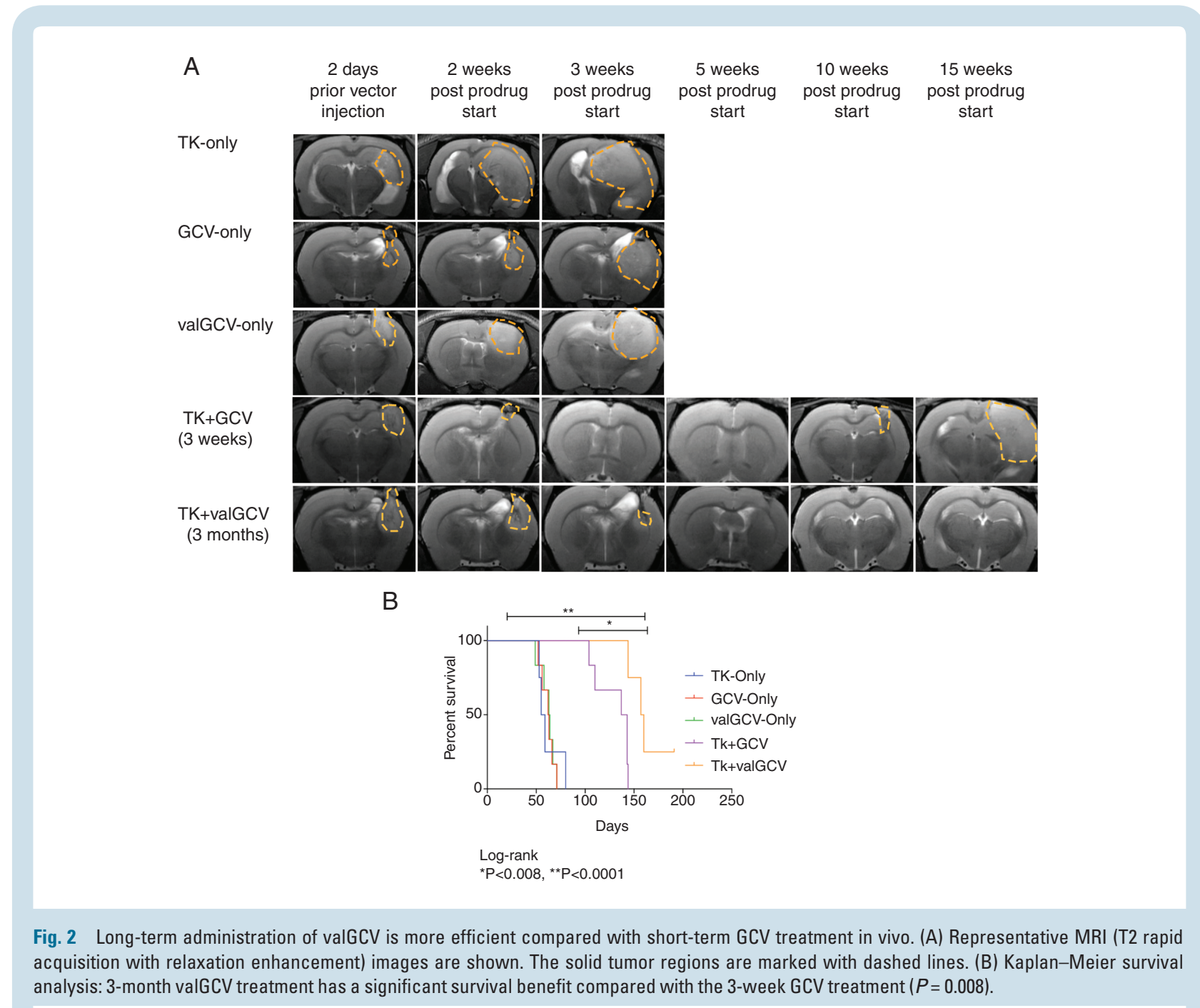


A

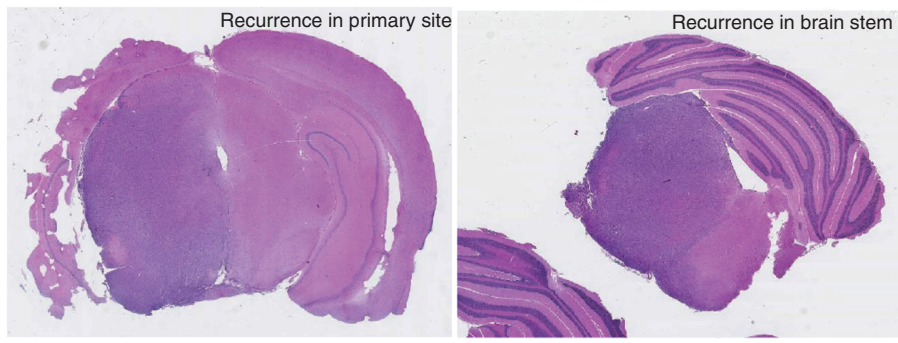

B

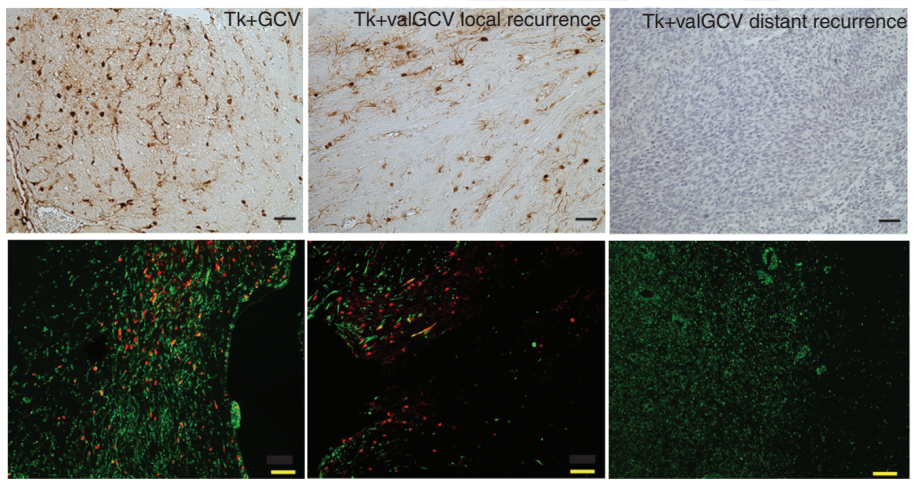

$\mathrm{D}$
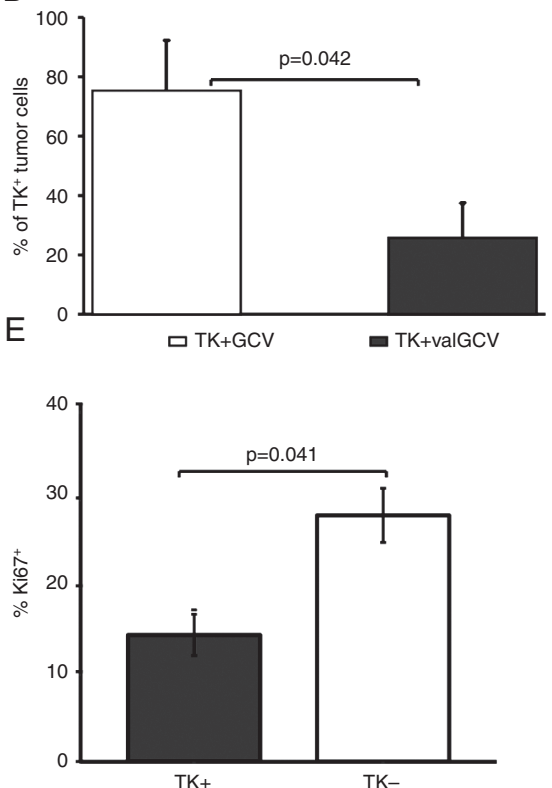

Fig. 3 Continuous valGCV application eliminates the majority of TK+ tumor cells. (A) Hematoxylin and eosin stainings of recurrences (left panel: recurrence at the primary site in GCV treatment group; right panel: recurrence distant from the primary site in valGCV treatment group). (B) Immunohistochemistry with antibodies for HSV-TK show transduced cells in the recurrent tumor mass. (C) Double immunofluorescent staining using antibodies against human nestin (green) and GFP (red) for the TK.007-eGFP fusion protein. Representative images show TK.007-eGFP+ tumor cells that survived prodrug treatment. Scale bar: $50 \mu \mathrm{m}$. (D) Quantification of the remaining tumor cells expressing TK.007-eGFP: percentage of residual TK+ tumor cells in 3-month-long valGCV-treated group is lower compared with the short-term GCV-treatment group $(P=0.042)$. (E) Proliferative capacity of the remaining TK+ cells is significantly lower compared with TK- cells $(P=0.041)$ in the TK + GCV group.

\section{Recurrent Tumors Are More Invasive and Less Angiogenic Compared with the Primary Tumors}

Histological analyses revealed that the recurrent tumors from both treatment groups were more invasive and showed reduced vascularization/angiogenesis compared with the primary tumors from the control groups (Fig. $4 \mathrm{~A}-\mathrm{F}$ ). Nestin immunohistochemistry revealed that GCVtreated tumors exhibited a diffuse growth pattern with extensive infiltration into neighboring anatomical structures such as corpus callosum and even the contralateral hemisphere (Fig. 4A). Quantification of number of invasive cells outside the solid tumor mass confirmed that GCV-treated tumors were significantly more invasive compared with control tumors, which both were found close to the primary injection site (Fig. 4B). All recurrent tumors of the valGCV group were found distant from the primary site, which confirms that these tumors were initiated by highly invasive cells that escaped the treatment. When analyzing angiogenic/hypoxic features, primary tumors showed highly abundant necrotic areas and characteristic microvascular proliferations, which both were absent in local and distant recurrences (Fig. 4C). Immunostaining for von Willebrand factor confirmed smaller vessels in the distant and local recurrent tumors compared with the primary tumors (Fig. 4D). Quantification of the vessel area fraction showed that the differences between primary and overall recurrent tumors (Fig. 4E), but not between the local and distant recurrences (Fig. 4F), were statistically significant.
Apparently, there was an emergence of invasive glioma cells that escaped prodrug treatment and established a secondary tumor mass mostly in brain regions distant from the primary site. Thus, we aimed at testing whether administration of valGCV in cycles (analogous to chemotherapy) might lower the threshold of surviving tumor cells for inducing an invasive escape program. We compared continuous valGCV treatment with cyclic valGCV using the experimental setup as described for Fig. 2. However, the cyclic administration scheme neither showed any improved therapeutic effect nor resulted in reduced infiltrative nature of the recurrent tumors (Supplementary Fig. 3A, B).

\section{Distant Tumor Recurrences Show Upregulation of EGFR and Downregulation of pSTAT3}

Due to the different growth pattern of distantly recurrent tumors in the valGCV group, we analyzed important molecular pathways involved in glioblastoma development by immunohistochemistry in order to identify proteins that might drive recurrent and invasive tumor growth. We performed immunostainings for (1) the tyrosine kinase receptors EGFR, platelet derived growth factor receptor A (PDGFR-A), and c-MET, which often are amplified or coamplified in GBM; (2) phosphorylated mitogen activated protein kinase (pMAPK) and phosphorylated signal transducer and activator of transcription 3 (pSTAT3), which are activated downstream of tyrosine kinase signaling; 


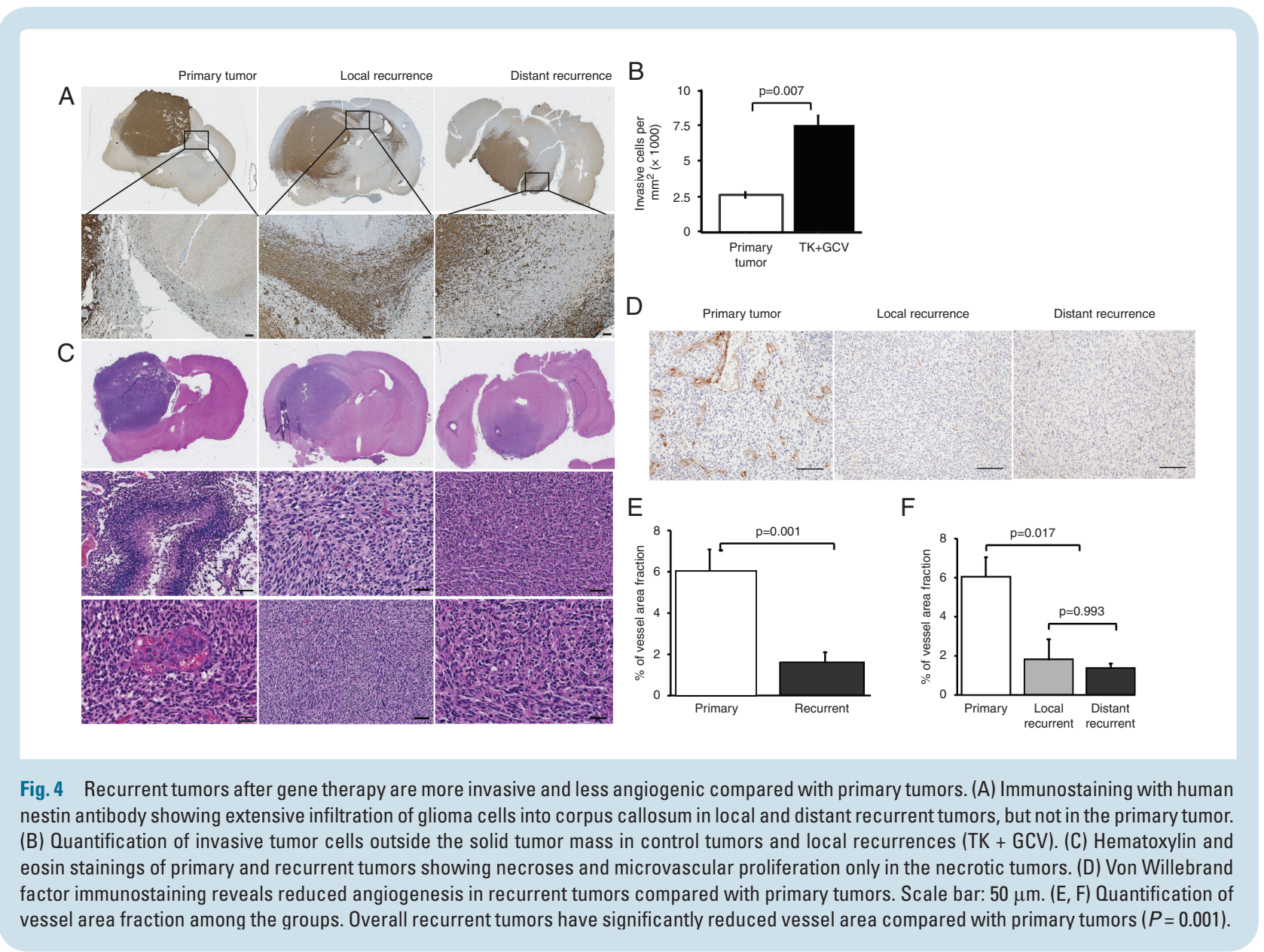

(3) olig2, a protein abundantly expressed in glial tumors (Fig. 5A, C, E and Supplementary Fig. 4A). Equally expressed in all groups without obvious differences were c-MET, olig2, and pMAPK. However, there was a striking difference in EGFR expression: While the primary tumors were negative for EGFR, we observed EGFR expression in the recurrent tumors (Fig. 5A). Quantification of EGFR immunohistochemistry revealed that recurrent tumors expressed significantly higher EGFR in general, and interestingly, distant recurrences further showed a significantly higher EGFR expression level compared with the local recurrences (Fig. 5B). To check if the EGFR upregulation involved amplification events, we performed fluorescence in situ hybridization for EGFR/chromosome (Chr) 7, which only showed Chr7 gain, but no amplification in both treatment and control groups (Supplementary Fig. 4B). In addition to EGFR upregulation, we found differential expression of PDGFR-A, which exhibited uniform and abundant expression in the recurrent tumor tissue, while it was very heterogeneously expressed in primary tumors with strong expression in the vessel wall, but weaker in the tumor tissue (Fig. 5C). Quantification revealed that there was a significant difference in PDGFR-A expression between primary versus recurrent tumors, but not between local versus distant recurrences (Fig. 5D). In contrast, pSTAT3 was significantly downregulated in recurrent tumors compared with primary tumors (Fig. 5E, F). However, these differential expression patterns did not affect proliferative activity, as shown by Ki-67 staining (Fig. 5G, H).

\section{Recurrent Tumors Are Resistant to Erlotinib Treatment}

Based on our observation that EGFR is upregulated in the recurrent tumors after gene therapy, we hypothesized that a combinatorial treatment regimen involving gene therapy plus anti-EGFR treatment would further improve the therapeutic benefit. To test this, we compared a combination therapy consisting of gene therapy plus erlotinib, a small-molecule EGFR inhibitor, with gene therapy alone. Resembling our previous animal experiments, tumor remission (on MRI) was observed within a 3-4 week time period in both treatment groups (data not shown) followed by tumor recurrences at later timepoints. Kaplan-Meier survival analysis showed that there was no survival difference between gene therapy only and combination therapy (Fig. 6A). To analyze the effect of erlotinib on EGFR phosphorylation, we immunostained brain sections of the different groups with an anti-pEGFR (1068) antibody (Fig. $6 \mathrm{~B})$. We observed a trend of reduced EGFR phosphorylation in the double treatment group compared with the gene therapy only group (Fig. 6C). We also immunostained brain sections with an anti-EGFR antibody, which showed 
A

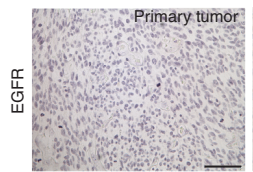

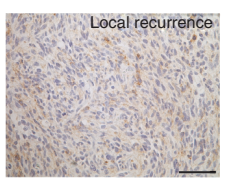

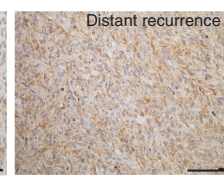

$\mathrm{E}$
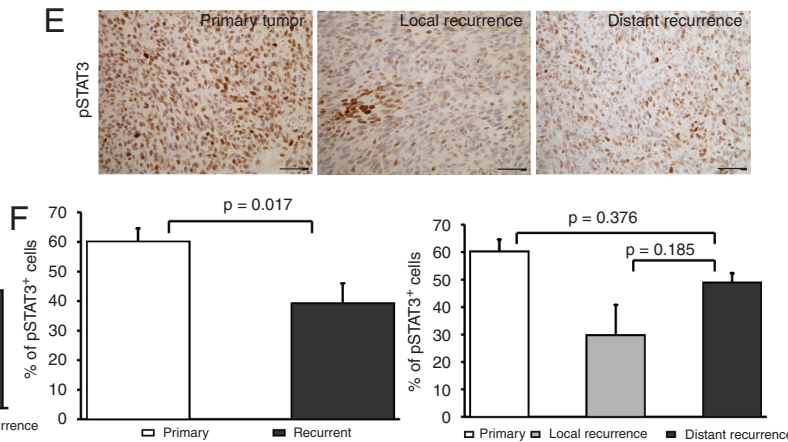

G

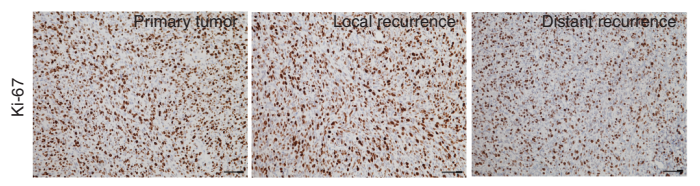

$\mathrm{D}$
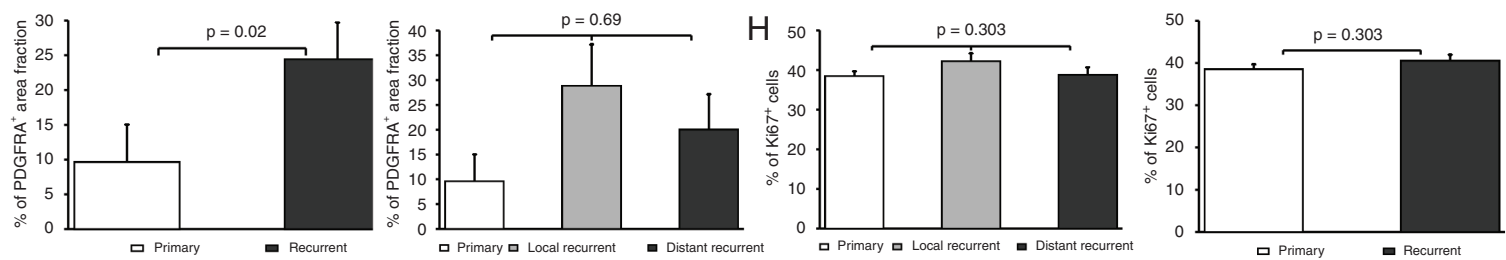

Fig. 5 EGFR is upregulated in recurrent tumors after suicide gene therapy. Immunohistochemical stainings with antibodies against EGFR (A), PDGFR-A (C), pSTAT3 (E), and Ki-67 (G). Quantification showing percentage of EGFR+ (B), PDGFR-A+ (D) area fraction, and pSTAT3+ cells (F). Scale bar: $50 \mu \mathrm{m}$. (H) Quantification of Ki-67+ cells; mean \pm SD.

significant upregulation of EGFR expression in the combination treatment group compared with the gene therapy only group (Fig. 6D, E). Thus, compensatory upregulation of EGFR in the tumor cells might impede the effect of antiEGFR treatment.

\section{Transcriptome Profiling of Recurrent Tumors After TK + valGCV Treatment}

To identify global transcriptional changes associated with tumor recurrence after TK + valGCV treatment, we performed RNA sequencing. Interspecies variability of the sequences showed equal contamination by host tissue among all samples except one which was excluded (Supplementary Fig. 5A). Gene expression differential analysis revealed 262 genes upregulated and 139 genes downregulated (adjusted $P<0.05$ ) when comparing the gene expression profiles between the TK + valGCV and valGCV only groups (Supplementary Fig. 5B, Supplementary Fig. 6A, B, Supplementary Table 1). Among the upregulated genes we detected EGFR (Supplementary Fig. 6B), confirming the previous results from immunohistochemistry in Fig. 5. Biological processes/pathways associated with the transcriptional changes were identified by gene set enrichment analyses. "Cellular response to hypoxia" and "vasculogenesis" were major processes associated with control tumors (Supplementary Fig. 6C, Supplementary Tables 2 and 3), confirming our previous results showing increased angiogenesis in this group (Fig. 4). Recurrent tumors from theTK + valGCV group exhibited upregulation of "Wnt signaling pathway" and "cell adhesion molecules," among others (Supplementary Fig. 6D, Supplementary Tables 2 and 3), indicating several invasive escape mechanisms in addition to EGFR upregulation.

\section{Discussion}

In suicide gene therapy of brain tumors, 1-2 weeks of prodrug treatment is a standard regimen that, by default, has been used in preclinical studies and clinical trials with replication-deficient vectors so far. $^{3,6,17,21-24}$ A similar prodrug administration scheme of a maximum of 2 weeks also dominates the ongoing clinical trials with replication-defective vectors (ClinicalTrials.gov identifiers: NCT03596086, NCT03603405, and NCT00634231). This regimen had been tested extensively in animal models and had shown success there. However, tumors in animal models proliferate at a higher rate compared with the human situation, and in particular animal models based on serum-cultured monolayer cell lines exhibit a highly proliferative and non-invasive behavior. ${ }^{25}$ The killing of tumor cells by GCV triphosphate (GCV-TP) is mediated in only proliferative cells, as GCV-TP is incorporated as a nucleotide-analogon into the host DNA of dividing cells 
A
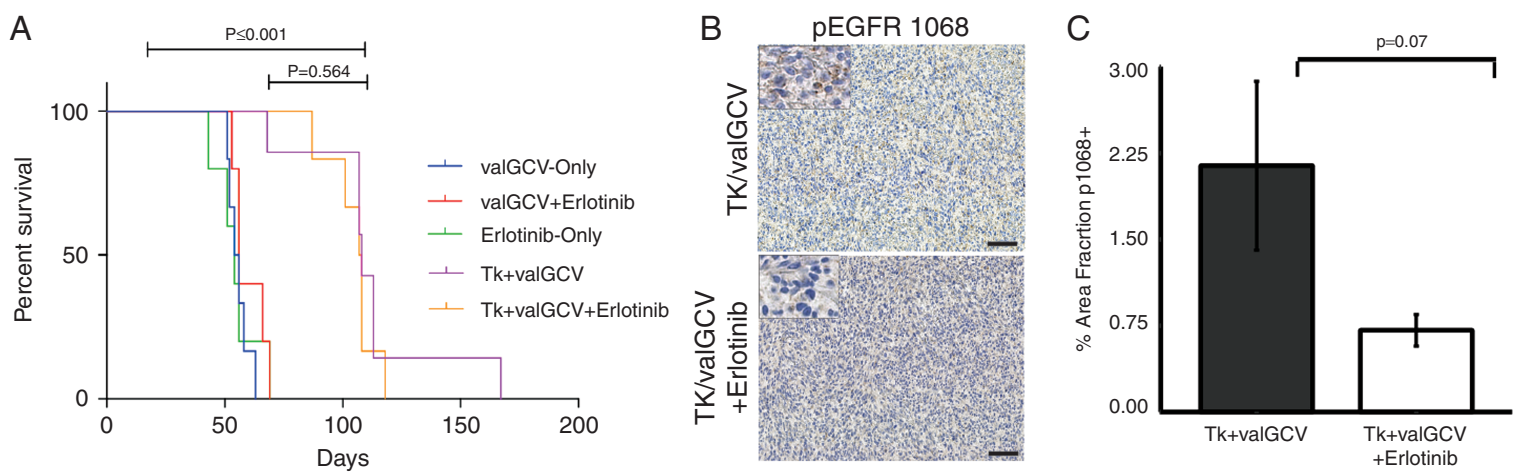

D

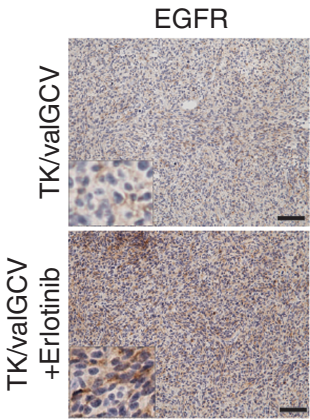

$\mathrm{E}$

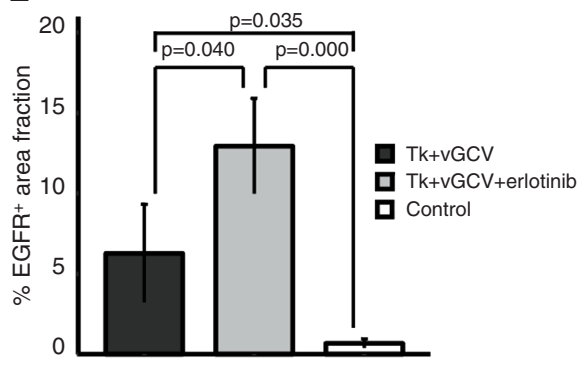

Fig. 6 Combination of suicide gene therapy with erlotinib does not improve therapeutic efficacy. (A) Kaplan-Meier survival analysis does not show a significant difference between double treatment and single treatment arms. (B) Immunohistochemical stainings with antibodies against pEGFR 1068. Scale bar: $50 \mu \mathrm{m}$. (C) Quantification showing percentage of pEGFR 1068+ area fraction. (D, E) Immunohistochemical stainings with antibodies against EGFR and quantification of EGFR+ area fraction.

and terminates DNA synthesis. Thus, tumor cells are killed much faster in highly proliferating tumors compared with slowly proliferating ones when using HSV-TK/GCV suicide gene therapy. For our study, we employed one of the most clinically relevant animal models for $\mathrm{GBM}^{12}$ that closely recapitulates patient histology upon orthotopic implantation, retains major genetic changes of patient GBM, and shows a similar proliferation rate. ${ }^{25}$ Importantly, this patient-derived xenograft (PDX) model shows profound heterogeneity at the single cell level and contains tumor cells from proneural, neural, classical, and mesenchymal subtypes (unpublished data). Using this model system, we previously showed that lentiviral-vector mediated HSV-TK/GCV gene therapy is a highly promising therapeutic option for GBM. ${ }^{8}$ However, a substantial fraction of $\mathrm{TK}+$ tumor cells were found to escape the standard GCV treatment. At that time, the mechanism of survival of residualTK-transduced tumor cells remained open. Genomic deletion or transgene silencing was described as one potential mechanism of TK resistance after retroviral-vector mediated delivery, ${ }^{26}$ but expression of the transgene is still detectable in transduced cells that survived treatment in our model. Aberrant transcription ${ }^{27}$ or splicing ${ }^{28}$ can result in expression of nonfunctional TK. However, TK.007 involves codon corrections and optimizations, which remove the cryptic sites in question. Moreover, the extensive codon optimization of TK.007 has removed many of the hotspots for mutations described for wild-type TK, ${ }^{29}$ although the generation of potential new sites prone to mutation could not be excluded.

However, we hypothesized that a fraction of the TK-expressing cells might become resistant through quiescence. In this work we showed that resistant TK+ glioma cells indeed were slowly proliferating in vivo and retained sensitivity to ganciclovir in vitro. These data supported our assumption that the standard treatment of 2-3 weeks might be not long enough to kill all (or at least the vast majority of) transduced cells. In turn, it was suggestive to propose that a prolonged scheme of prodrug administration should result in better therapeutic outcome. However, systemic GCV treatment is associated with various drawbacks including cathether infections and thus is not a suitable option for long-term administration. ${ }^{18}$ Instead, we decided to use valGCV as a prodrug, which is established as a preemptive treatment regimen in cytomegalovirus disease and has been documented to be safe and well tolerated by GBM patients after administration for more than 6 months. ${ }^{19}$ ValGCV is tailored as an oral formulation that readily gets metabolized to the active form (ganciclovir) in the intestinal wall and the liver. ${ }^{30}$ Due to its similar efficacy compared with intravenous GCV, significantly higher bioavailability than oral GCV, and safer mode of administration than intravenous GCV, valGCV has become a standard drug in clinical practice for cytomegalovirus infection. ${ }^{18}$

In line with our hypothesis, we observed a significant survival benefit of the prolonged valGCV treatment 
regimen and significant reduction of $\mathrm{TK}+$ cells compared with GCV short-term treatment. However, except for one rat in the valGCV group, all other rats demonstrated tumor recurrence at various timepoints after initial tumor remission. Interestingly, the recurrence patterns between GCV and valGCV groups were spatially different. The distant recurrences of the valGCV-treated animals lacked tumor cells with TK.007-eGFP expression, suggesting that highly invasive tumor cells that had not been transduced with TK escaped the treatment. Histological, transcriptomic, and immunohistochemical analyses confirmed that recurrent tumors were more invasive and less angiogenic compared with the primary tumors, while the primary tumors involved abundant necrotic areas and microvascular proliferation. Enhanced invasion of recurrent GBMs was also observed in other therapeutic settings, such as bevacizumab treatment most likely induced by hypoxia. ${ }^{31-33}$ In our setting, intermittent hypoxia could also play a role, since lentiviral vectors can transduce endothelial cells, ${ }^{34}$ and killing these cells through HSV-TK/GCV could lead to less vascularization and hypoxia. Thus, similar mechanisms could be involved in inducing tumor invasion in different therapeutic settings.

Molecular characterization of the recurrent tumors by RNA sequencing and immunohistochemical analyses revealed upregulation of EGFR. The level of expression was also significantly higher in distant compared with local recurrences. EGFR was previously associated with tumor invasion in many different cancer types, ${ }^{35-38}$ and we previously showed that EGFR mediates invasion of tumor cells independently of angiogenesis. ${ }^{13,39}$ Keunen et al showed upregulation of EGF in bevacizumab-treated GBM xenografts, indicating that hypoxia and a lack of blood supply could be a potential mechanism for EGFR upregulation/activation. ${ }^{32}$ Thus, EGFR appeared to be an attractive target for a combinatorial treatment setting with suicide gene therapy to prevent tumor recurrence. Erlotinib is a prominent EGFR inhibitor that can cross the BBB ${ }^{40-42}$ and downregulates EGFR activation (phosphorylation). There was a trend of reduced activation of EGFR upon erlotinib administration, but it was neither significant nor translated into therapeutic benefit (and/or reduced invasion of tumor cells). Inhibition of EGFR phosphorylation was reported to trigger various adaptive responses and thereby can resist therapy. ${ }^{43-45}$ In line, single inhibition of EGFR by erlotinib was shown to be ineffective in a glioma PDX model unless combined with other inhibitors to target the corresponding associated proteins that drive reactive compensatory mechanisms. ${ }^{44}$ It is noteworthy in this context that gene enrichment analysis of RNA sequencing data revealed upregulation of additional genes/pathways in the TK + valGCV treated tumors, including Wnt signaling, which is known to be an important player in glioma invasion. ${ }^{46}$

In conclusion, we show that the prodrug administration scheme is a key factor for suicide gene therapy of GBM. We report that long-term treatment with the prodrug valGCV improves HSV-TK suicide gene therapy for GBM by killing the tumor cells that still retain sensitivity toward the prodrug but are not eliminated by the standard short-term GCV treatment. Based on our results, prolonged and continuous administration of prodrug should be considered for suicide gene therapy studies and/or clinical trials for brain tumors in the future.

\section{Supplementary Material}

Supplementary data are available at Neuro-Oncology online.

\section{Keywords}

brain tumors | EGFR | glioblastoma | lentiviral vectors | suicide gene therapy

\section{Funding}

This work was supported by the Helse Vest, Haukeland University Hospital, Research Council of Norway, the Norwegian Cancer Society, the K.G. Jebsen Research Foundation, and the Bergen Medical Research Foundation. J. A. Hossain and M. A. Latif were supported by Post-Doc and PhD fellowships respectively from Helse Vest.

\section{Acknowledgments}

We thank B. Nordanger, I. Gavlen, T. Johansen, H. S. Sdik, B. B Tysnes, and B. Hansen for expert technical assistance and the Molecular Imaging Center (MIC) in Bergen, Norway for technical support.

Conflict of interest statement. The authors declare that they have no conflict of interests.

Authorship statement. JAH and HM performed experiments and wrote the manuscript. LARY, MAL, SN, JVJ, KMT, and JG performed experiments. KR and BF provided lentiviral vectors and wrote the manuscript. AM, MAL, and FA performed bioinformatics analysis. $\mathrm{RB}$ wrote the manuscript.

\section{References}

1. Stupp R, Mason WP, van den Bent MJ, et al; European Organisation for Research and Treatment of Cancer Brain Tumor and Radiotherapy Groups; National Cancer Institute of Canada Clinical Trials Group. Radiotherapy plus concomitant and adjuvant temozolomide for glioblastoma. N Engl J Med. 2005;352(10):987-996.

2. Patel AP, Tirosh I, Trombetta JJ, et al. Single-cell RNA-seq highlights intratumoral heterogeneity in primary glioblastoma. Science. 2014;344(6190):1396-1401.

3. Ram Z, Culver KW, Oshiro EM, et al. Therapy of malignant brain tumors by intratumoral implantation of retroviral vector-producing cells. Nat Med. 1997;3(12):1354-1361. 
4. Shand N, Weber F, Mariani L, et al. A phase 1-2 clinical trial of gene therapy for recurrent glioblastoma multiforme by tumor transduction with the herpes simplex thymidine kinase gene followed by ganciclovir. GLI328 European-Canadian Study Group. Hum Gene Ther. 1999;10(14):2325-2335.

5. Rainov NG, Ren H. Clinical trials with retrovirus mediated gene therapy—what have we learned? J Neurooncol. 2003;65(3):227-236.

6. Rainov NG. A phase III clinical evaluation of herpes simplex virus type 1 thymidine kinase and ganciclovir gene therapy as an adjuvant to surgical resection and radiation in adults with previously untreated glioblastoma multiforme. Hum Gene Ther. 2000; 11(17):2389-2401.

7. Naldini L, Blömer U, Gallay $P$, et al. In vivo gene delivery and stable transduction of nondividing cells by a lentiviral vector. Science. 1996;272(5259):263-267.

8. Huszthy PC, Giroglou T, Tsinkalovsky 0, et al. Remission of invasive, cancer stem-like glioblastoma xenografts using lentiviral vectormediated suicide gene therapy. PLoS One. 2009;4(7):e6314.

9. Hossain JA, Ystaas LR, Mrdalj J, et al. Lentiviral HSV-Tk.007-mediated suicide gene therapy is not toxic for normal brain cells. J Gene Med. 2016;18(9):234-243.

10. Miletic H, Fischer YH, Giroglou T, et al. Normal brain cells contribute to the bystander effect in suicide gene therapy of malignant glioma. Clin Cancer Res. 2007;13(22 Pt 1):6761-6768.

11. Sakariassen PØ, Prestegarden L, Wang J, et al. Angiogenesisindependent tumor growth mediated by stem-like cancer cells. Proc Natl Acad Sci U S A. 2006;103(44):16466-16471.

12. Bjerkvig R, Tønnesen A, Laerum OD, Backlund EO. Multicellular tumor spheroids from human gliomas maintained in organ culture. J Neurosurg. 1990;72(3):463-475.

13. Eskilsson E, Rosland GV, Talasila KM, et al. EGFRvIll mutations can emerge as late and heterogenous events in glioblastoma development and promote angiogenesis through Src activation. Neuro Oncol. 2016;18(12):1644-1655.

14. Hossain JA, Riecken K, Miletic H, Fehse B. Cancer suicide gene therapy with TK.007. Methods Mol Biol. 2019;1895:11-26.

15. Preuss E, Muik A, Weber K, Otte J, von Laer D, Fehse B. Cancer suicide gene therapy with TK.007: superior killing efficiency and bystander effect. J Mol Med (Berl). 2011;89(11):1113-1124.

16. Preuss E, Treschow A, Newrzela S, et al. TK.007: a novel, codonoptimized HSVtk(A168H) mutant for suicide gene therapy. Hum Gene Ther. 2010;21(8):929-941.

17. Westphal M, Ylä-Herttuala S, Martin J, et al; ASPECT Study Group. Adenovirus-mediated gene therapy with sitimagene ceradenovec followed by intravenous ganciclovir for patients with operable highgrade glioma (ASPECT): a randomised, open-label, phase 3 trial. Lancet Oncol. 2013;14(9):823-833.

18. Biron KK. Antiviral drugs for cytomegalovirus diseases. Antiviral Research. 2006; 71(2-3):154-163.

19. Stragliotto G, Rahbar A, Solberg NW, et al. Effects of valganciclovir as an add-on therapy in patients with cytomegalovirus-positive glioblastoma: a randomized, double-blind, hypothesis-generating study. Int J Cancer. 2013;133(5):1204-1213.

20. O'Grady NP, Alexander M, Burns LA, et al; Healthcare Infection Control Practices Advisory Committee (HICPAC). Guidelines for the prevention of intravascular catheter-related infections. Clin Infect Dis. 2011;52(9):e162-e193.

21. Ram Z, Culver KW, Walbridge S, Blaese RM, Oldfield EH. In situ retroviral-mediated gene transfer for the treatment of brain tumors in rats. Cancer Res. 1993;53(1):83-88.

22. Klatzmann D, Valéry CA, Bensimon G, et al. A phase $\mathrm{I} / \mathrm{Il}$ study of herpes simplex virus type 1 thymidine kinase "suicide" gene therapy for recurrent glioblastoma. Study Group on Gene Therapy for Glioblastoma. Hum Gene Ther. 1998;9(17):2595-2604.
23. King GD, Muhammad AK, Curtin JF, et al. Flt3L and TK gene therapy eradicate multifocal glioma in a syngeneic glioblastoma model. Neuro Oncol. 2008;10(1):19-31.

24. Immonen A, Vapalahti M, Tyynelä K, et al. AdvHSV-tk gene therapy with intravenous ganciclovir improves survival in human malignant glioma: a randomised, controlled study. Mol Ther. 2004;10(5):967-972.

25. Huszthy PC, Daphu I, Niclou SP, et al. In vivo models of primary brain tumors: pitfalls and perspectives. Neuro Oncol. 2012;14(8):979-993.

26. Frank 0, Rudolph C, Heberlein C, et al. Tumor cells escape suicide gene therapy by genetic and epigenetic instability. Blood. 2004;104(12):3543-3549.

27. Salomon B, Maury S, Loubière L, Caruso M, Onclercq R, Klatzmann D. A truncated herpes simplex virus thymidine kinase phosphorylates thymidine and nucleoside analogs and does not cause sterility in transgenic mice. Mol Cell Biol. 1995;15(10):5322-5328.

28. Chalmers D, Ferrand C, Apperley JF, et al. Elimination of the truncated message from the herpes simplex virus thymidine kinase suicide gene. Mol Ther. 2001;4(2):146-148.

29. Frobert E, Ooka T, Cortay JC, Lina B, Thouvenot D, Morfin F. Herpes simplex virus thymidine kinase mutations associated with resistance to acyclovir: a site-directed mutagenesis study. Antimicrob Agents Chemother. 2005:49(3):1055-1059.

30. Cvetković RS, Wellington K. Valganciclovir: a review of its use in the management of CMV infection and disease in immunocompromised patients. Drugs. 2005;65(6):859-878.

31. de Groot JF, Fuller G, Kumar AJ, et al. Tumor invasion after treatment of glioblastoma with bevacizumab: radiographic and pathologic correlation in humans and mice. Neuro Oncol. 2010;12(3):233-242.

32. Keunen 0 , Johansson $M$, Oudin $A$, et al. Anti-VEGF treatment reduces blood supply and increases tumor cell invasion in glioblastoma. Proc Natl Acad Sci U S A. 2011;108(9):3749-3754.

33. Pàez-Ribes $M$, Allen $E$, Hudock $J$, et al. Antiangiogenic therapy elicits malignant progression of tumors to increased local invasion and distant metastasis. Cancer Cell. 2009;15(3):220-231.

34. Totsugawa $T$, Kobayashi $N$, Maruyama $M$, et al. Lentiviral vector: a useful tool for transduction of human liver endothelial cells. ASAIO J. 2003;49(6):635-640.

35. Radinsky R, Risin S, Fan D, et al. Level and function of epidermal growth factor receptor predict the metastatic potential of human colon carcinoma cells. Clin Cancer Res. 1995;1(1):19-31.

36. Liu Y, Ji R, Li J, et al. Correlation effect of EGFR and CXCR4 and CCR7 chemokine receptors in predicting breast cancer metastasis and prognosis. J Exp Clin Cancer Res. 2010;29:16.

37. Bos PD, Zhang XH, Nadal $\mathrm{C}$, et al. Genes that mediate breast cancer metastasis to the brain. Nature. 2009;459(7249):1005-1009.

38. Okada Y, Hurwitz EE, Esposito JM, Brower MA, Nutt CL, Louis DN. Selection pressures of TP53 mutation and microenvironmental location influence epidermal growth factor receptor gene amplification in human glioblastomas. Cancer Res. 2003;63(2):413-416.

39. Talasila KM, Soentgerath A, Euskirchen P, et al. EGFR wild-type amplification and activation promote invasion and development of glioblastoma independent of angiogenesis. Acta Neuropathol. 2013;125(5):683-698.

40. Togashi $Y$, Masago K, Fukudo M, et al. Cerebrospinal fluid concentration of erlotinib and its active metabolite OSI-420 in patients with central nervous system metastases of non-small cell lung cancer. J Thorac Oncol. 2010;5(7):950-955.

41. Katayama T, Shimizu J, Suda K, et al. Efficacy of erlotinib for brain and leptomeningeal metastases in patients with lung adenocarcinoma who showed initial good response to gefitinib. J Thorac Oncol. 2009:4(11):1415-1419.

42. Broniscer A, Panetta JC, O'Shaughnessy M, et al. Plasma and cerebrospinal fluid pharmacokinetics of erlotinib and its active metabolite OSI-420. Clin Cancer Res. 2007;13(5):1511-1515. 
43. Zhang $Z$, Lee JC, Lin $L$, et al. Activation of the AXL kinase causes resistance to EGFR-targeted therapy in lung cancer. Nat Genet. 2012;44(8):852-860.

44. Guo G, Gong K, Ali S, et al. A TNF-JNK-Axl-ERK signaling axis mediates primary resistance to EGFR inhibition in glioblastoma. Nat Neurosci. 2017;20(8):1074-1084.
45. Akhavan D, Pourzia AL, Nourian AA, et al. De-repression of PDGFR $\beta$ transcription promotes acquired resistance to EGFR tyrosine kinase inhibitors in glioblastoma patients. Cancer Discov. 2013;3(5):534-547.

46. Lee Y, Lee JK, Ahn SH, Lee J, Nam DH. WNT signaling in glioblastoma and therapeutic opportunities. Lab Invest. 2016;96(2):137-150. 